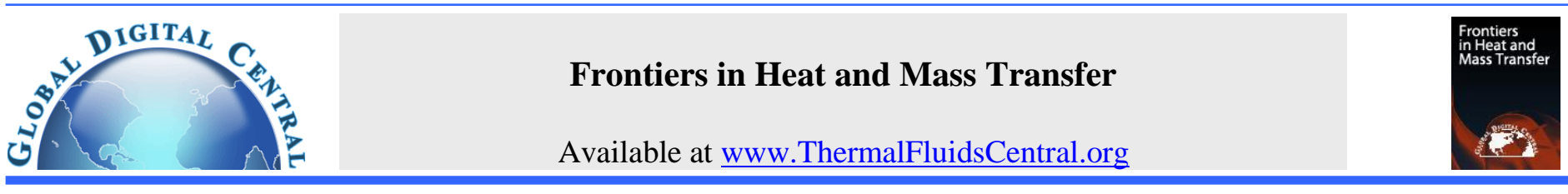

\title{
PERFORMANCE OF THE OPTIMIZED TREE-TYPE CYLINDRICAL- SHAPED NANOPOROUS FILTERING MEMBRANES WITH 9 OR 10 BRANCH PORES IN EACH PORE TREE
}

\author{
Yongbin Zhang* \\ College of Mechanical Engineering, Changzhou University, Changzhou, Jiangsu Province, China
}

\begin{abstract}
The paper analytically studies the performance of the optimized tree-type cylindrical-shaped nanoporous filtering membranes with 9 or 10 branch pores in each pore tree. The optimum ratio of the radius of the trunk pore to the radius of its branch pore was found. The corresponding lowest flow resistances of the membranes were typically calculated respectively for weak, medium and strong liquid-pore wall interactions. For liquid-liquid separations, the optimum radii of the trunk pore in the membranes were calculated according to the weak liquid-pore wall interaction. The capability of the liquidliquid separation of the membranes was also studied. The obtained results are of significance to the design of these membranes.
\end{abstract}

Keywords: Membrane; Nanopore; Filtration; Separation; Optimization

\section{INTRODUCTION}

Nanoporous filtering membranes are a new direction of the development of filtration membranes due to their super purification capability (Adiga et al., 2009; Hinds et al., 2004). They have been successfully applied in ordinary and medical purifications (Baker and Bird, 2008; Biffinger et al., 2007; Fissel et al., 2009; Jackson and Hillmyer, 2010). One of the challenges to the development of these membranes is that it is not easily reconciled among the filtration capability, flux and mechanical strength of the membranes. In the past, it was tried to overcome it such as by manufacturing very thin membranes with the use of the material of grapheme, taking the filtration pore as conical, and taking the membrane as mixed and consisted of both nanopores and micropores (Li et al., 2004; Surwade et al., 2015; Yang et al., 2006).

The author proposed an optimized cylindrical-shaped nanoporous filtering membrane across the thickness of which are manufactured two concentric pores with different radii (Zhang, 2018a). The smaller pore is for filtration with nanometer scale radius and pore depth, while the radius of the other pore is optimized for achieving the lowest flow resistance of the membrane. Besides the filtration capability, both the flux and the mechanical strength of this membrane are substantially improved.

Later, a tree-type cylindrical-shaped nanoporous filtering membrane was proposed for overcoming the above mentioned challenge (Zhang, 2018b). This membrane can be further optimized by taking the branch pore as parallel with its trunk pore and optimizing the radius of the trunk pore for yielding the lowest flow resistance of the membrane (Zhang, 2018c). It has been found that the increase of the number $\mathrm{N}$ of the branch pores in each pore tree results in a significant reduction of the flow resistance of the membrane when $\mathrm{N}$ is no more than 10; However, when $\mathrm{N}$ is over 10 , the increase of $\mathrm{N}$ cannot result in a further pronounced reduction of the flow resistance of the membrane, but on the other hand it will cause more complicated manufacturing problems. It seems that from the viewpoint of an engineering application, the maximum value of $\mathrm{N}$ should be 10 .
For the purpose of application, the present paper aims to provide an analysis for the performance of the optimized tree-type cylindricalshaped nanoporous filtering membrane with 9 or 10 branch pores in each pore tree. The optimum ratio of the radius of the trunk pore to the radius of its branch pore was found. The corresponding lowest flow resistances of the membranes were typically calculated respectively for weak, medium and strong liquid-pore wall interactions. For liquid-liquid separations, the optimum radii of the trunk pore in the membranes were calculated according to the weak liquid-pore wall interaction for widely varying operational parameter values. The obtained results are important for the design of the mentioned membranes.

\section{STUDIED MEMBRANES}

Figures 1(a), (b) and (c) show the studied tree-type cylindrical-shaped nanoporous filtering membranes with 9 or 10 branch pores in each pore tree. The pore trees are evenly distributed within the membrane. In each pore tree, there are $\mathrm{N}$ equal branch pores respectively for filtration with the radius $R_{b, 1}$ on the $1 \mathrm{~nm}$ or $10 \mathrm{~nm}$ scales and with the pore depth $l_{1}$, there is one trunk pore with the pore depth $l_{2}$ and with the radius $R_{b, 2}$ usually on the $10 \mathrm{~nm}$ or $100 \mathrm{~nm}$ scales which is however optimized for achieving the lowest flow resistance of the membrane, and the branch pore is parallel with its trunk pore. The physical properties of the surfaces of all the pores are identical. The thickness of the membrane is $l=\left(l_{1}+l_{2}\right)$.

\section{ANALYSIS}

The analysis for the flow within the studied membranes is based on the flow equation for a nanoscale fluid flow. It was presented in Ref.12 in details. Here are only presented the necessary contents.

According to the analysis, the $\mathrm{N}$ branch pores in each pore tree is equivalent to the straight cylindrical pore with the radius, which is solved from the following equation (Zhang, 2018c): 


$$
\frac{N \cdot C q\left(\bar{R}_{b, 1}\right)\left|S\left(\bar{R}_{b, 1}\right)\right| \bar{R}_{b, 1}^{4}}{C y\left(\bar{R}_{b, 1}\right)}-\frac{C q\left(\bar{R}_{e q}\right)\left|S\left(\bar{R}_{e q}\right)\right| \bar{R}_{e q}^{4}}{C y\left(\bar{R}_{e q}\right)}=0
$$

where $\bar{R}_{b, 1}=R_{b, 1} / R_{c r}, \bar{R}_{e q}=R_{e q} / R_{c r}, R_{c r}$ is the critical radius of the pore for the passing liquid to become continuum across the pore radius, $C y(\bar{R})=\eta_{b f}^{\text {eff }}(\bar{R}) / \eta, \quad C q(\bar{R})=\rho_{b f}^{\text {eff }}(\bar{R}) / \rho, \quad \rho_{b f}^{\text {eff }} \quad$ and $\eta_{b f}^{\text {eff }}$ are respectively the average density and the effective viscosity of the passing liquid across the pore radius, $S$ is the parameter describing the noncontinuum effect of the passing liquid across the pore radius

$(-1 \leq S<1)$, and $\rho$ and $\eta$ are respectively the bulk density and the bulk viscosity of the passing liquid at the environmental temperature and pressure.
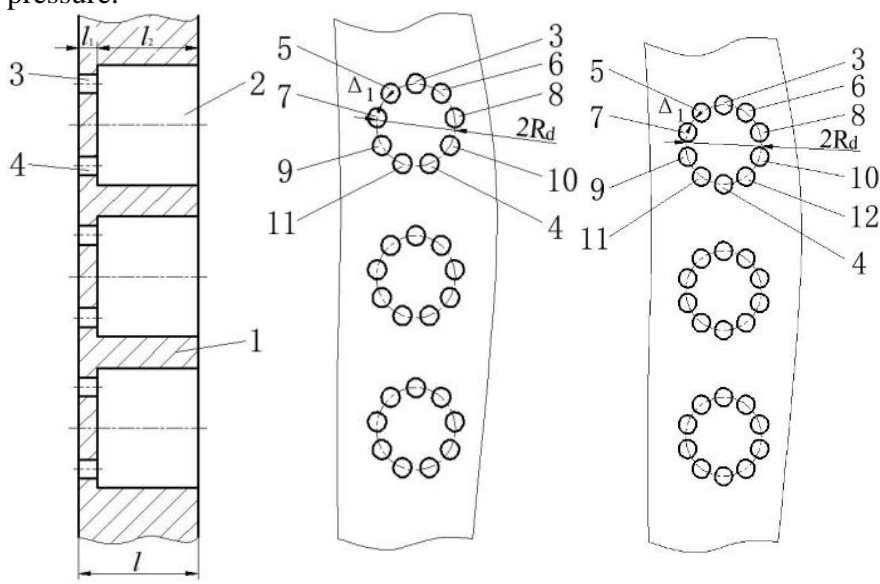

(a) Front view

$\begin{array}{lll}\text { (b) Left-side view, } N=9 & \text { (c) Left-side view, } N=10\end{array}$ 1-Membrane substrate; 2-Trunk pore; 3,4,5,6,7,8,9,10,11,12-Branch pores

Fig. 1 The optimized tree-type cylindrical-shaped nanoporous filtering membranes with 9 or 10 branch pores in each pore tree studied in the present paper (with $N=9$ or 10) (Zhang, 2018c).

For yielding the lowest flow resistance of the membrane, there is the optimum ratio of the radius $R_{b, 2}$ of the trunk pore to the radius $R_{b, 1}$ of the branch pore which should be calculated as: $\left(R_{b, 2} / R_{b, 1}\right)_{\text {opt }}=$ $\left(R_{e q} / R_{b, 1}\right)\left(R_{b, 2} / R_{e q}\right)_{o p t}$. Here, the optimum ratio of $R_{b, 2}$ to $R_{e q}$ is calculated as (Zhang, 2018c):

$\left(\frac{R_{b, 2}}{R_{e q}}\right)_{o p t}= \begin{cases}{\left[\frac{\left(1-\lambda_{0}\right) C q\left(\bar{R}_{e q}\right)\left|S\left(\bar{R}_{e q}\right)\right|}{\lambda_{0} C y\left(\bar{R}_{e q}\right)}\right]^{\frac{1}{4}}} & \quad \text { for } R_{b, 2} \geq R_{e q} \\ 1 \quad & \text { for } R_{b, 2}<R_{e q}\end{cases}$

where $\lambda_{0}=l_{1} / l$.

For the optimum value of $R_{b, 2}$, the corresponding dimensionless lowest flow resistance of the membrane is calculated as: $I_{f, \min }=$ $\left(\bar{R}_{r} / \bar{R}_{e q}\right)^{2} F_{\text {min }}$, where $\bar{R}_{r}=R_{r} / R_{c r}, R_{r}$ is a constant reference radius, and $F_{\min }$ is (Zhang, 2018c):

$$
F_{\min }= \begin{cases}2 \sqrt{\frac{\lambda_{0}\left(1-\lambda_{0}\right) C y\left(\bar{R}_{e q}\right)}{C q\left(\bar{R}_{e q}\right)\left|S\left(\bar{R}_{e q}\right)\right|}} & \text { for } R_{b, 2} \geq R_{e q} \\ \frac{C y\left(\bar{R}_{e q}\right)}{C q\left(\bar{R}_{e q}\right)\left|S\left(\bar{R}_{e q}\right)\right|} & \text { for } R_{b, 2}<R_{e q}\end{cases}
$$

In liquid-liquid separations, the optimum value of $R_{b, 2}$ should be determined to yield the lowest flow resistance of the membrane for the liquid which has a weak interaction with the pore wall. For the other mixed liquids which may respectively have medium or strong interactions with the pore wall, the dimensionless flow resistance of the membrane should be calculated as: $I_{f}=\left(\bar{R}_{r} / \bar{R}_{e q}\right)^{2} F\left(R_{b, 2} / R_{e q}\right)$, where $F\left(R_{b, 2} / R_{e q}\right)$ is (Zhang, 2018c):

$F\left(\frac{R_{b, 2}}{R_{e q}}\right)=\frac{\lambda_{0} C y\left(\bar{R}_{e q}\right)}{C q\left(\bar{R}_{e q}\right)\left|S\left(\bar{R}_{e q}\right)\right|}\left(\frac{R_{b, 2}}{R_{e q}}\right)^{2}+\frac{\left(1-\lambda_{0}\right) C y\left(\bar{R}_{b, 2}\right)}{C q\left(\bar{R}_{b, 2}\right)\left|S\left(\bar{R}_{b, 2}\right)\right|\left(\frac{R_{b, 2}}{R_{e q}}\right)^{2}}$

where $\bar{R}_{b, 2}=R_{b, 2} / R_{c r}$.

\section{CALCUlation}

For studying the performance of the membranes in Fig.1 in liquidparticle separations, the optimum ratios $\left(R_{b, 2} / R_{b, 1}\right)_{\text {opt }}$ and the corresponding dimensionless lowest flow resistance $I_{f, \min }$ of the membrane were typically calculated respectively for weak, medium and strong liquid-pore wall interactions when $R_{b, 1}$ and $\lambda_{0}$ were widely varied. For studying the performance of the membranes in Fig. 1 in liquidliquid separations, the optimum values of the radius $R_{b, 2}$ of the trunk pore were calculated according to the weak liquid-pore wall interaction for varying $R_{b, 1}$ and $\lambda_{0}$; For this determined optimum $R_{b, 2}$, the dimensionless flow resistances $I_{f}$ of the membranes were typically calculated for the mixed liquids which may respectively have weak, medium and strong interactions with the pore wall, when $R_{b, 1}$ varies from $0.5 \mathrm{~nm}$ to $20 \mathrm{~nm}$. In the calculations, it was taken that $R_{r}=10 \mathrm{~nm}$.

In the calculations, for whichever liquid-pore wall interaction, $C q(\bar{R})$ is generally expressed as (Zhang, 2018c):

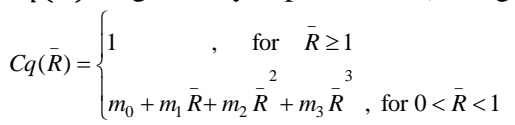

where $\bar{R}$ is $\bar{R}_{b, 1}, \bar{R}_{e q}$ or $\bar{R}_{b, 2}$ (same in the following equations), $m_{0}$, $m_{1}, m_{2}$ and $m_{3}$ are respectively constants.

$\operatorname{Cy}(\bar{R})$ is generally expressed as (Zhang, 2018c):

$C y(\bar{R})=\left\{\begin{array}{lll}1 \quad & \text { for } \quad \bar{R} \geq 1 \\ a_{0}+\frac{a_{1}}{\bar{R}}+\frac{a_{2}}{\bar{R}^{2}} & , & \text { for } 0<\bar{R}<1\end{array}\right.$

where $a_{0}, a_{1}$ and $a_{2}$ are respectively constants.

$S(\bar{R})$ is generally expressed as (Zhang, 2018c):

$S(\bar{R})= \begin{cases}-1 \quad, & \text { for } \quad \bar{R} \geq 1 \\ {\left[n_{0}+n_{1}\left(\bar{R}-n_{3}\right)^{n_{2}}\right]^{-1}} & , \text { for } n_{3}<\bar{R}<1\end{cases}$

where $n_{0}, n_{1}, n_{2}$ and $n_{3}$ are respectively constants.

For weak, medium and strong liquid-pore wall interactions, the values of $R_{c r}$ were respectively taken as $3.5 \mathrm{~nm}, 10 \mathrm{~nm}$ and $20 \mathrm{~nm}$ (Zhang, 2018c). For different types of the liquid-pore wall interaction, the values of the other parameters are respectively shown in Tables 1(ac).

Table 1(a) Liquid viscosity data for different liquid-pore wall interaction types (Zhang, 2018c)

\begin{tabular}{|l|l|l|l|}
\hline Interaction & \multicolumn{1}{|c|}{$\mathrm{a}_{0}$} & \multicolumn{1}{c|}{$\mathrm{a}_{1}$} & $\mathrm{a}_{2}$ \\
\hline Strong & & & \\
\hline Medium & 1.8335 & -1.4252 & 0.5917 \\
\hline Weak & 1.0822 & -0.1758 & 0.0936 \\
\hline
\end{tabular}

Table 1(b) Liquid density data for different liquid-pore wall interaction types (Zhang, 2018c)

\begin{tabular}{|l|l|c|c|c|}
\hline Interaction & \multicolumn{1}{|c|}{$\mathrm{m}_{0}$} & $\mathrm{~m}_{1}$ & $\mathrm{~m}_{2}$ & $\mathrm{~m}_{3}$ \\
\hline Strong & & & & \\
\hline Medium & 1.43 & -1.723 & 2.641 & -1.347 \\
\hline Weak & 1.30 & -1.065 & 1.336 & -0.571 \\
\hline
\end{tabular}


Table 1(c) Liquid non-continuum property data for different liquidpore wall interaction types (Zhang, 2018c)

\begin{tabular}{|l|l|c|c|c|}
\hline Interaction & \multicolumn{1}{|c|}{$\mathrm{n}_{0}$} & $\mathrm{n}_{1}$ & $\mathrm{n}_{2}$ & $\mathrm{n}_{3}$ \\
& & & & \\
\hline Strong & 0.4 & -1.374 & -0.534 & 0.035 \\
\hline Medium & -0.649 & -0.343 & -0.665 & 0.035 \\
\hline Weak & -0.1 & -0.892 & -0.084 & 0.1 \\
\hline
\end{tabular}

\section{RESULTS AND DISCUSSIONS}

Figure 2(a) shows the values of the optimum ratios $\left(R_{b, 2} / R_{b, 1}\right)_{\text {opt }}$ respectively for the weak, medium and strong liquid-pore wall interactions when $\lambda_{0}=1 \times 10^{-3}$ and $N=9$ or 10 . The values of $\left(R_{b, 2} / R_{b, 1}\right)_{\text {opt }}$ are obviously different respectively for $N=9$ and $N=10$ for the other same operating conditions. Figure 2(b) shows the corresponding dimensionless lowest flow resistances $I_{f, \min }$ of the membranes respectively for the weak, medium and strong liquid-pore wall interactions when $R_{r}=10 \mathrm{~nm}$. For the same liquid-pore wall interaction, in Fig.2(b), the curve for $N=9$ is nearly overlaid with that for $N=10$. For the same operating conditions, there is little difference between the resulting lowest flow resistances of the membranes respectively for $N=9$ and $N=10$ when the ratio $\left(l_{1}+l_{2}\right)$ is optimum.

Figure 3(a) plots the optimum values of the ratio $\left(l_{1}+l_{2}\right)$ against $\lambda_{0}$ respectively for the weak, medium and strong liquid-pore wall interactions when $\bar{R}_{b, 1}=0.5$ and $N=9$ or 10 . For a given $\lambda_{0}$ which is no more than $1 \times 10^{-3}$ and the same liquid-pore wall interaction, the optimum value of $\left(l_{1}+l_{2}\right)$ for $N=10$ is obviously greater than that for $N=9$. However, for $\lambda_{0}>1 \times 10^{-3}$, there are little differences between the optimum values of $R_{b, 2} / R_{b, 1}$ respectively for $N=9$ and $N=10$ when the liquid-pore wall interactions are the same. For a given $\mathrm{N}$ and the given liquid-pore wall interaction, the optimum value of $\left(l_{1}+l_{2}\right)$ is significantly increased with the reduction of $\lambda_{0}$ especially when $\lambda_{0}$ is low.

Figure 3(b) plots the corresponding dimensionless lowest flow resistance $I_{f, \min }$ of the membranes against $\lambda_{0}$ respectively for the weak, medium and strong liquid-pore wall interactions when $R_{r}=$ $10 \mathrm{~nm}, \bar{R}_{b, 1}=0.5$, and $N=9$ or 10 . For a given $\lambda_{0}$ and the same liquidpore wall interaction, the value of $I_{f, \min }$ for $N=10$ is a little lower than that for $N=9$. The value of $I_{f, \min }$ is significantly reduced with the reduction of $\lambda_{0}$ for any $N$ and any liquid-pore wall interaction. Figure 3(b) shows that for given $\lambda_{0}$ and $N$, the value of $I_{f, \min }$ is significantly reduced with the increase of the strength of the interaction between the liquid and the pore wall. This is due to the actually significantly different values of the dimensional radius $R_{b, 1}$ of the branch pore for different liquid-pore wall interactions in the condition of $\bar{R}_{b, 1}=0.5$, as the critical radii $R_{c r}$ are significantly different for different liquid-pore wall interactions as shown in Section 4.

Figure 4 shows the optimum values of the radius $R_{b, 2}$ of the trunk pore of the membranes determined according to the weak liquid-pore wall interaction for different $R_{b, 1}$ and $\lambda_{0}$. The membranes designed by these optimum $R_{b, 2}$ values can be used for liquid-liquid separations where one liquid has a weak interaction with the pore wall while the other liquids have medium or strong interactions with the pore wall.

Figure 5 typically plots the dimensionless flow resistances of the membranes against the radius $R_{b, 1}$ of the branch pore for Liquid A, Liquid B and Liquid C, which respectively have the weak, medium and strong interactions with the pore wall, when the radius $R_{b, 2}$ of the trunk pore is optimized for achieving the lowest flow resistances of the membranes for Liquid A as shown in Fig.4. It is shown that the radius $R_{b, 1}$ of the branch pore should be no more than $3 \mathrm{~nm}$ so that the flow resistance of the membrane for Liquid $\mathrm{C}$ i.e. the strong liquid-pore wall interaction is more than 10 times that for Liquid $\mathrm{A}$ i.e. the weak liquid- pore wall interaction in the same operating conditions. Further lower values of $R_{b, 1}$ give better performances of the membranes for liquidliquid separations, as the difference between the flow resistances of the membranes for different liquids in the same operating conditions is further rapidly enlarged. There is little difference between the performances of the membranes respectively for $N=9$ and $N=10$ for liquid-liquid separations, as the curves for these membranes in Fig.5 for the same liquids are all overlaid. The performance of the liquid-liquid separation of the membranes is shown to be obviously dependent on the differences among the interactions of the mixed liquids with the pore wall.

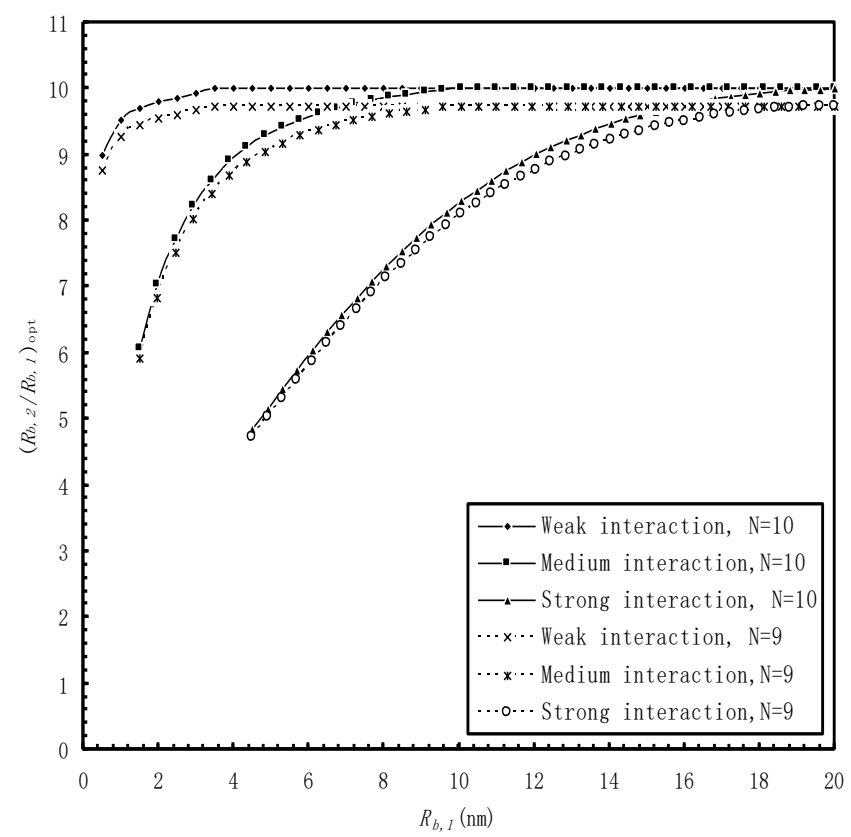

(a)

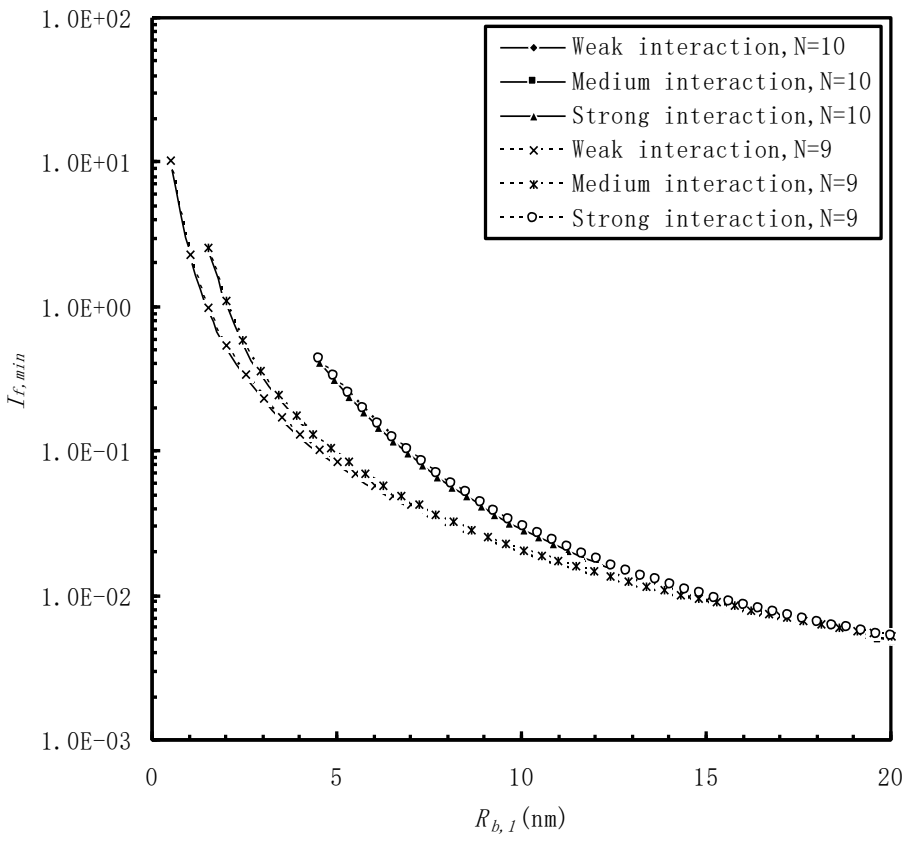

(b)

Fig. 2 Optimum values of the ratio $\left(l_{1}+l_{2}\right)$ and the corresponding dimensionless lowest flow resistances $I_{f, \min }$ of the membranes respectively for the weak, medium and strong liquid-pore wall interactions when $R_{r}=10 \mathrm{~nm}, \lambda_{0}=1 \times 10^{-3}$, and $N=9$ or 10 . 


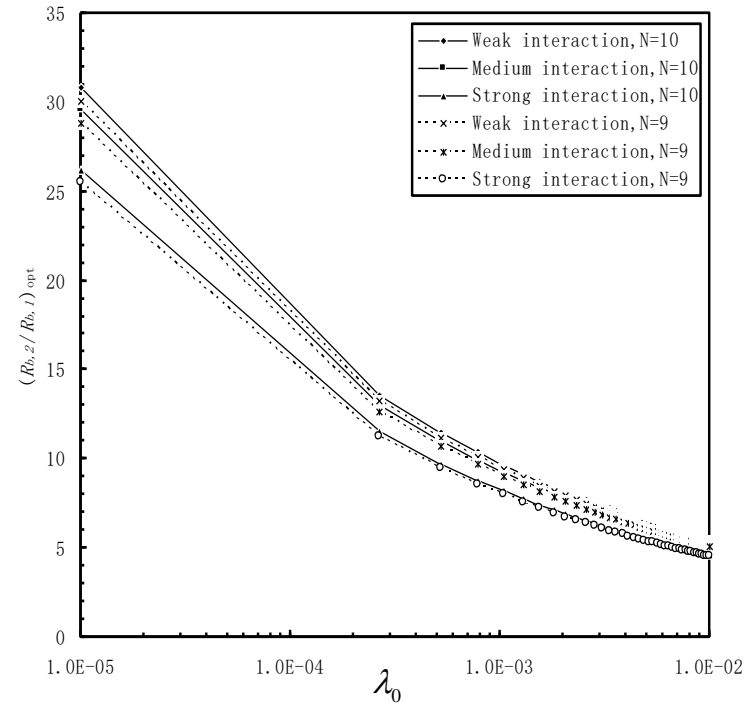

(a)

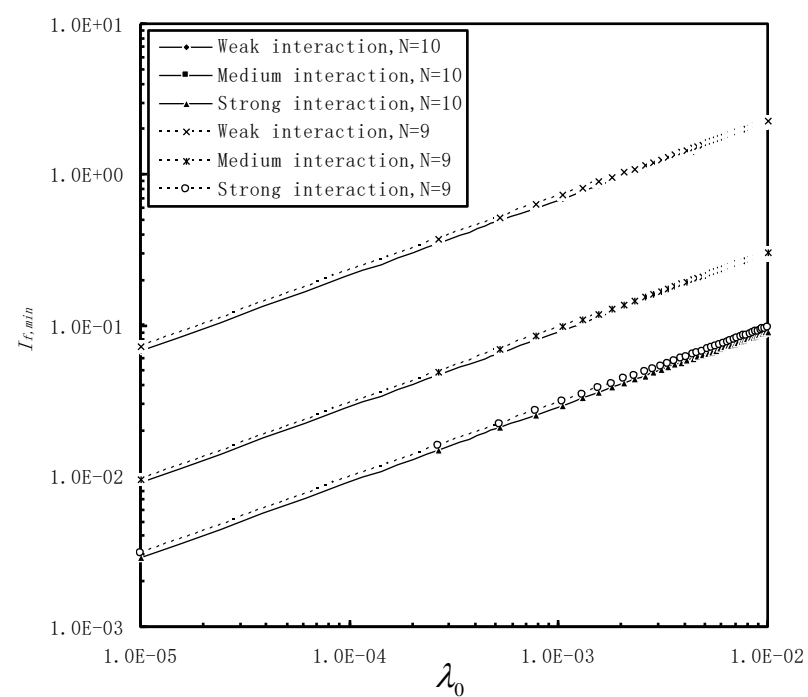

(b)

Fig. 3 Optimum values of the ratio $\left(l_{1}+l_{2}\right)$ and the corresponding dimensionless lowest flow resistances $I_{f, \min }$ of the membranes respectively for the weak, medium and strong liquid-pore wall interactions when $R_{r}=10 \mathrm{~nm}, \bar{R}_{b, 1}=0.5$, and $N=9$ or 10 .

\section{CONCLUSIONS}

The performances of the optimized tree-type cylindrical-shaped nanoporous filtering membranes are analytically investigated when the number $N$ of the branch pores in each pore tree is respectively 9 and 10 . For yielding the lowest flow resistances of these membranes, the optimum values of the ratio of the radius $R_{b, 2}$ of the trunk pore to the radius $R_{b, 1}$ of the branch pore were typically calculated respectively for weak, medium and strong liquid-pore wall interactions for $R_{b, 1}$ ranging between $0.5 \mathrm{~nm}$ and $20 \mathrm{~nm}$; The corresponding dimensionless lowest flow resistances of these membranes were also calculated.

For the purpose of liquid-liquid separations, the optimum values of $R_{b, 2}$ in these membranes were calculated according to the weak liquidpore wall interaction for widely varying operational parameter values. The capability of the liquid-liquid separation of these membranes was investigated based on the calculated optimum $R_{b, 2}$ values. It was found that the performance of these membranes in liquid-liquid separation is mainly dependent on both the radius $R_{b, 1}$ of the branch pore and the difference among the interactions of the mixed liquids with the pore wall; In the same operating condition, there is little difference between the performances of the membranes respectively for $N=9$ and $N=10$ in liquidliquid separations. The obtained results in this paper are of significant interest to the engineering design and application of the proposed membranes with $N=9$ or 10 .

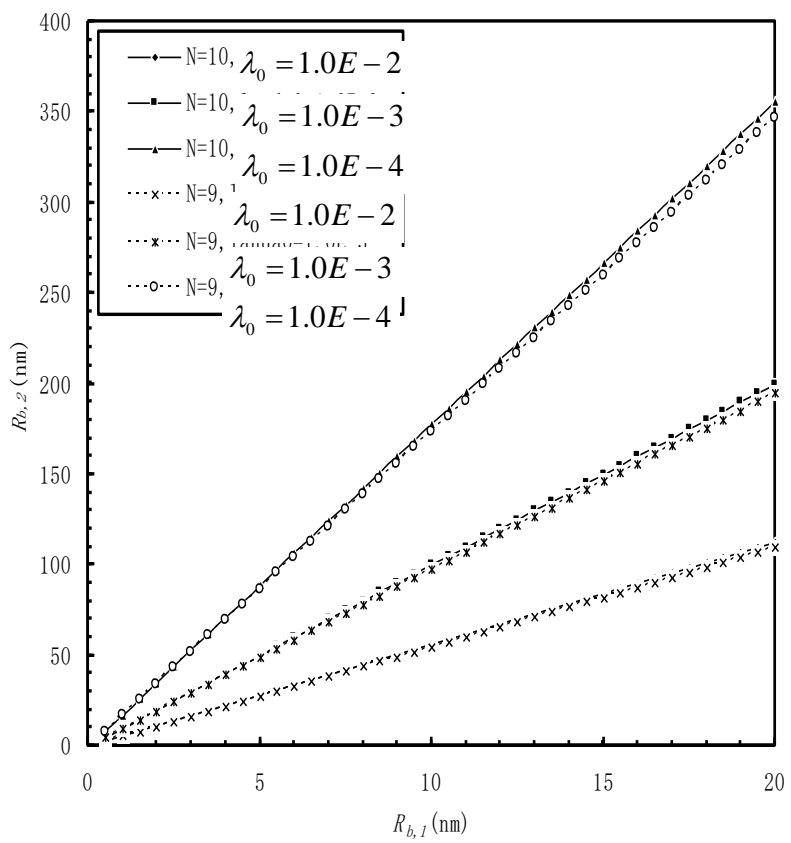

Fig. 4 Optimum values of the radius $R_{b, 2}$ of the trunk pore of the membranes determined according to the weak liquid-pore wall interaction.

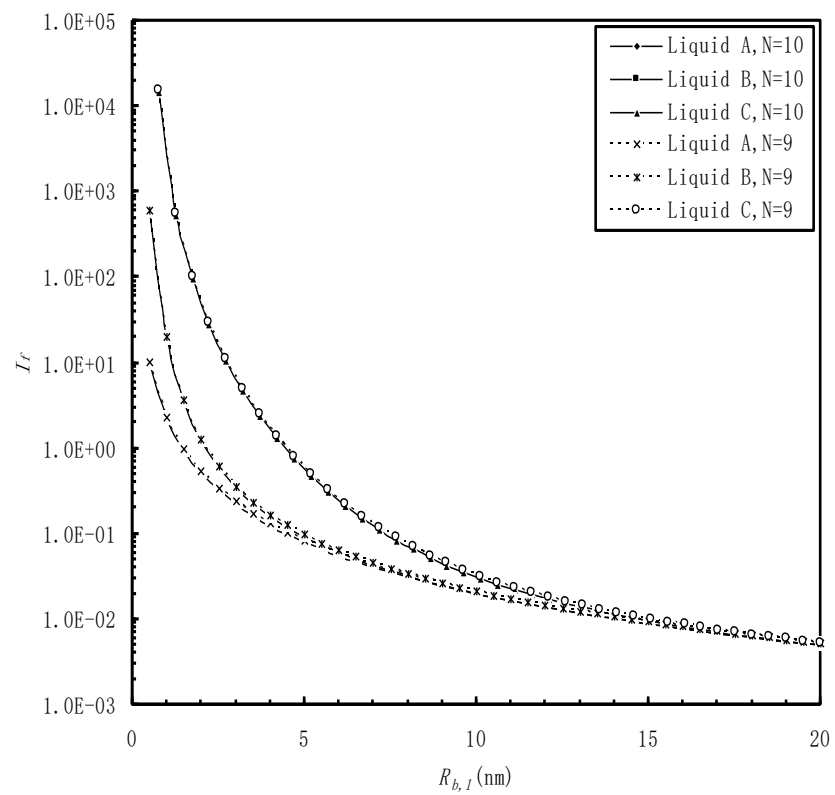

Fig. 5 Plots of the dimensionless flow resistances of the membranes against the radius $R_{b, 1}$ of the branch pore for Liquid $\mathrm{A}$, Liquid $\mathrm{B}$ and Liquid $\mathrm{C}$ when $\lambda_{0}=1 \times 10^{-3}$ and the radius $R_{b, 2}$ of the trunk pore is optimized according to Liquid A as shown in Fig.4 
For the purpose of liquid-liquid separations, the optimum values of $R_{b, 2}$ in these membranes were calculated according to the weak liquidpore wall interaction for widely varying operational parameter values. The capability of the liquid-liquid separation of these membranes was investigated based on the calculated optimum $R_{b, 2}$ values. It was found that the performance of these membranes in liquid-liquid separation is mainly dependent on both the radius $R_{b, 1}$ of the branch pore and the difference among the interactions of the mixed liquids with the pore wall; In the same operating condition, there is little difference between the performances of the membranes respectively for $N=9$ and $N=10$ in liquidliquid separations. The obtained results in this paper are of significant interest to the engineering design and application of the proposed membranes with $N=9$ or 10 .

\section{REFERENCES}

Adiga, S. P., Jin, C., Curtiss, L. A., Monteiro-Riviere, N. A., and Narayan, R. J., 2009, "Nanoporous Membranes for Medical and Biological Applications," Nanomedicine and Nanobiotechnology, 1, 568-581. https://doi.org/10.1002/wnan.50

Baker, L. A., and Bird, S. P., 2008, "Nanopores: A Makeover for Membranes," Nature Nanotechnology, 3, 73-74. https://doi.org/10.1038/nnano.2008.13

Biffinger, J. C., Ray, R., Little, B., and Ringeisen, B. R., 2007, "Diversifying Biological Fuel Cell Designs by Use of Nanoporous Filters," Environmental Science and Technology, 41, 1444-1449. https://doi.org/10.1021/es061634u

Fissel, W. H., Dubnisheva, A., Eldridge, A. N., Fleischman, A. J., Zydney, A. L., and Roy, S., 2009, "High-Performance Silicon Nanopore Hemofiltration Membranes," Journal of Membrane Science, 326, 58-63. https://doi.org/10.1016/j.memsci.2008.09.039
Hinds, B. J., Chopra, N., Rantell, T., Andrews, R., Gavalas, M. V., and Bachas, L. G., 2004, "Aligned Multiwalled Carbon Nanotube Membranes," Science, 303, 62-65. https://doi.org/10.1126/science.1092048

Jackson, E. A., and Hillmyer, M. A., 2010, "Nanoporous Membranes Derived From Block Copolymers: From Drug Delivery to Water Filtration," Acs Nano, 4, 3548-3553. https://doi.org/10.1021/nn1014006

Li, N., Yu, S., Harrell, C., and Martin, C. R., 2004, "Conical Nanopore Membranes: Preparation and Transport Properties," Analytical Chemistry, 76, 2025-2030. https://doi.org/10.1021/ac035402e

Surwade, S. P., Smirnov, S. N., Vlassiou, I. V., Unocic, R. R., Veith, G. M., Dai, S., and Mahurin, S. M., 2015, "Water Desalination Using Nanoporous Single-Layer Graphene," Nature Nanotechnology, 10, 459464.

https://doi.org/10.1038/nnano.2015.37

Yang, S. Y., Ryu, I., Kim, H., Kim, J., Jang, S., and Russell, T., 2010, "Nanoporous Membranes with Ultrahigh Selectivity and Flux for the Filtration of Viruses," Advanced Materials, 18, 709-712. https://doi.org/10.1002/adma.200501500

Zhang, Y. B., 2018a, "Optimum Design for Cylindrical-Shaped Nanoporous Filtration Membrane," International Communications in Heat and Mass Transfer, 96, 130-138. https://doi.org/10.1016/j.icheatmasstransfer.2018.06.003.

Zhang, Y. B., 2018b, “A Tree-Type Cylindrical-Shaped Nanoporous Filtering Membrane," Frontiers in Heat and Mass Transfer, 10, 16. http://dx.doi.org/10.5098/hmt.10.16

Zhang, Y. B., 2018c, "An Optimized Tree-Type Cylindrical-Shaped Nanoporous Filtering Membrane," Frontiers in Heat and Mass Transfer, 11, 25. http://dx.doi.org/10.5098/hmt.11.25 